\title{
Trends in the prevalence of elevated skinfold thickness among children and adolescents in Shandong Province, China, 1995-2014
}

\author{
Shu-Rong Wang ${ }^{1, *}$, Yan Cheng ${ }^{1}$, Min Chen ${ }^{1}$ and Ying-Xiu Zhang ${ }^{2, *}$ \\ 'Shandong Blood Center, 22 Shanshi East Road, Jinan, Shandong 250014, People's Republic of China: ${ }^{2}$ Shandong \\ Center for Disease Control and Prevention, Shandong University Institute of Preventive Medicine, 16992 Jingshi \\ Road, Jinan, Shandong 250014, People's Republic of China
}

Submitted 23 August 2017: Final revision received 19 December 2017: Accepted 6 March 2018: First published online 4 April 2018

\begin{abstract}
Objective: There are abundant data on secular trends in BMI; however, information on the change in subcutaneous fat is limited. The present study examined the trends in the prevalence of elevated skinfold thickness among children and adolescents over the past 19 years (1995-2014) in Shandong Province, China.

Design: Data for the study were obtained from two cross-sectional surveys of schoolchildren carried out in 1995 and 2014. Triceps and subscapular skinfold thicknesses (SFT) of all participants were measured. The sum of triceps and subscapular SFT (SSFT) was applied. Children and adolescents with SSFT above or equal to the national age- and sex-specific 85th percentile were defined as 'high SFT'.

Setting: Shandong Province, China.

Subjects: A total of 16917 students aged 7-18 years were included.

Results: For both boys and girls, an increasing trend in 'high SFT' was observed between 1995 and 2014. The overall prevalence of 'high SFT' increased from $10.31 \%$ for boys and $13.40 \%$ for girls in 1995 to $33.94 \%$ for boys and $29.30 \%$ for girls in $2014(P<0 \cdot 01)$.

Conclusions: There are more children and adolescents with very high levels of SFT. These trends describe very unfavourable changes in the body composition and should give cause for concern.
\end{abstract}

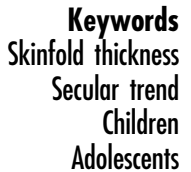

Childhood obesity has increased dramatically during the past decades, both in developing and developed countries $^{(1,2)}$. In 2009-2010, $18.2 \%$ of US children and adolescents aged $6-19$ years were obese ${ }^{(3)}$. As a populous country, it was estimated that $5 \cdot 1 \%$ of Chinese children and adolescents aged 7-18 years were obese in $2010^{(4)}$. Therefore, the worldwide prevalence of obesity in children and adolescents, with its attendant health risks, has become an important public health concern ${ }^{(5,6)}$.

BMI is perhaps the most commonly used measure for defining overweight and obesity in clinical practice and population surveys. However, BMI cannot distinguish between fat and fat-free mass; it provides no information on body fat distribution. Several studies have shown that compared with BMI, the amount and distribution of body fat play a greater role in the development of obesity-related complications such as CVD and type 2 diabetes ${ }^{(7)}$. A number of different tools and methodologies have been developed to measure body composition, of which skinfold thicknesses (SFT) have been shown to be closely correlated with total body fatness ${ }^{(8-10)}$. Furthermore, SFT measurements are non-invasive, simple and less expensive than laboratory-based techniques, so they have been widely applied in large-scale epidemiological studies.

Shandong Province, located in the lower reaches of the Yellow River, facing Japan and the Korean Peninsula to the east, is an important littoral province in East China. Secular trends in BMI and the prevalence of obesity among children and adolescents in this region have been documented in previous studies ${ }^{(11,12)}$. In the present article, we report the trends in the prevalence of elevated SFT among children and adolescents over the last 19 years (1995-2014) in Shandong, China.

\section{Participants and methods}

The study was approved by the Ethical Committee of the Shandong Center for Disease Control and Prevention, Shandong, China. 


\section{Study population}

Data for the present study were obtained from two national surveys on students' constitution and health carried out by the government in 1995 and 2014 in Shandong Province, China. A total of 16917 students, aged 7-18 years, of Han nationality in Shandong Province were included in the study (7198 in 1995 and 9719 in 2014). The sample size of age groups of each survey is given in Table 1. There were 299 to 427 participants in each of the age and sex groups in different years. The sampling method was stratified multistage sampling based on socio-economic status, drawn from three cities* as survey districts, and using randomly selected primary and secondary schools, the sample proportions in these three districts in each survey were equal. Six public schools (two primary schools, two junior high schools and two senior high schools) from each of the three districts in Shandong were randomly selected and invited to participate in the survey. From the selected schools, two classes in each grade were selected and all students of the selected classes were invited to join the study with informed consents. All participants were primary- and secondary-school students, ranging from 7 to 18 years of age, and all were of Han ancestry that accounts for $\sim 99.32 \%$ of the total population in Shandong. Most importantly, the schools from which the participants were sampled were relatively stable since 1995 , and the method and quality control of measurements of the two surveys was the same.

\section{Measurements}

All measurements were performed by well-trained health professionals in each of the three districts using the same type of apparatus and following the same procedures. SFT were measured on the right side of the body using a skinfold calliper (Minjian model GMCS-PZQ; Beijing Xindong Huateng Sports Instruments Company, Limited) to the nearest $0.5 \mathrm{~mm}$, at two sites: (i) triceps, halfway between the acromion process and the olecranon process; and (ii) subscapular, $1.0 \mathrm{~cm}$ below the inferior angle of the scapula, at an angle of $45^{\circ}$ to the lateral side of the body. In each participant, three measurements were taken, and the middle value was recorded for one skinfold site. The sum of triceps and subscapular SFT (SSFT) was applied.

\section{Statistical analyses}

Children and adolescents with SSFT above or equal to the national age- and sex-specific 85th percentile (13) $^{\text {were }}$ defined as 'high SFT'. Prevalence rates of 'high SFT' were

\footnotetext{
* The three cities were: (i) Jinan, the capital and the political, economic and cultural centre of Shandong Province, which has an area of $8227 \mathrm{~km}^{2}$ and a population of 7.07 million in 2014 , the GDP (gross domestic product) per capita was \$US 13409 in 2014; (ii) Yantai, an eastern coastal and developed city, with an area of $13739 \mathrm{~km}^{2}$ and a population of 7.00 million in 2014, people's living standards and seafood intake are relatively higher, the GDP per capita was \$US 14021 in 2014; and (iii) Jining, a western inland developing city with an area of $11000 \mathrm{~km}^{2}$ and a population of 8.24 million in 2014, people's living standards are relatively lower, the GDP per capita was \$US 7552 in 2014
}

determined, with comparisons between 1995 and 2014 made by the $\chi^{2}$ test. All analyses were performed with the statistical software package SPSS version 11.5. Significance was defined at the 0.05 level.

\section{Results}

The prevalence of 'high SFT' among children and adolescents by gender and age categories in 1995 and 2014 is shown in Table 1 . The $\chi^{2}$ test indicated that the prevalence of 'high SFT' surveyed in 2014 was significantly higher than that in 1995 in all age groups $(P<0.05$ or 0.01$)$. The overall prevalence of 'high SFT' increased from 10.31\% (boys) and $13.40 \% \%$ (girls) in 1995 to $33.94 \%$ (boys) and $29.30 \%$ (girls) in $2014(P<0.01)$. The prevalence of 'high SFT' by gender and age stage is shown in Fig. 1. During the 19-year period, for children (aged 7-12 years), the prevalence of 'high SFT' increased by 27.79 (boys) and 23.71 (girls) percentage points (from $9.78 \%$ for boys and $13 \cdot 17 \%$ for girls in 1995 to $37 \cdot 57 \%$ for boys and $36 \cdot 88 \%$ for girls in 2014), while for adolescents (aged 13-18 years), 'high SFT' prevalence increased by 19.45 (boys) and 8.06 (girls) percentage points (from $10.83 \%$ for boys and $13.62 \%$ for girls in 1995 to $30 \cdot 28 \%$ for boys and $21.68 \%$ for girls in 2014), respectively, indicating that the increment was bigger in children than in adolescents.

\section{Discussion}

To the best of our knowledge, the present study is the first examining the trends in the prevalence of elevated SFT among children and adolescents over the last 19 years in Shandong, one of the populous provinces in China. We found that there are more children and adolescents with very high levels of SFT; these trends describe very unfavourable changes in the body composition and should give cause for concern.

Body fat increases when energy intake is consistently greater than energy expenditure. In China, the rapid economic growth has led to changes in dietary and physical activity patterns, including an increase in the consumption of energy-dense foods and a decrease in physical activity ${ }^{(14,15)}$. These changes in lifestyle may play an important role in the observed increases in SFT reported here. Our results are in accordance with findings from other studies ${ }^{(16)}$.

In the current study, we found that the prevalence of 'high SFT' among children and adolescents had a rapid increase in the past 19 years, supporting other analyses based on BMI, which show a rapid increase in the prevalence of overweight and obesity ${ }^{(4,11,12)}$. The increasing prevalence of childhood overweight and obesity may be attributed to a markedly changed lifestyle associated with inactivity and dietary habits. Chinese diets have changed vastly in the last decades. The traditional diet, in which plant foods were given priority, has been replaced by the 
Table 1 Prevalence of 'high SFT' (sum of triceps and subscapular skinfold thickness above or equal to the national age- and sex-specific 85th percentile) among children and adolescents aged 7-18 years, Shandong Province, China ( $n 16$ 917), in 1995 and 2014

\begin{tabular}{|c|c|c|c|c|c|c|c|c|}
\hline \multirow[b]{3}{*}{ Age (years) } & \multicolumn{4}{|c|}{1995} & \multicolumn{4}{|c|}{2014} \\
\hline & \multicolumn{2}{|c|}{ Boys } & \multicolumn{2}{|c|}{ Girls } & \multicolumn{2}{|c|}{ Boys } & \multicolumn{2}{|c|}{ Girls } \\
\hline & $n$ & $\%$ & $n$ & $\%$ & $n$ & $\%$ & $n$ & $\%$ \\
\hline $7 \cdot 0-7.9$ & 300 & $9 \cdot 33$ & 300 & 13.00 & 419 & $37 \cdot 23^{\star \star}$ & 427 & $35 \cdot 13^{\star \star}$ \\
\hline $8.0-8.9$ & 300 & 8.33 & 300 & 10.00 & 406 & $40.39^{\star \star}$ & 371 & $38.54^{\star *}$ \\
\hline $9 \cdot 0-9 \cdot 9$ & 300 & 9.67 & 300 & $12 \cdot 33$ & 422 & $42 \cdot 89^{\star \star}$ & 412 & $39.08^{\star \star}$ \\
\hline $10 \cdot 0-10.9$ & 300 & 9.33 & 300 & 11.67 & 413 & $39.95^{\star \star}$ & 405 & $36.79^{\star \star}$ \\
\hline $11.0-11.9$ & 300 & 9.00 & 299 & $15 \cdot 38$ & 416 & $34 \cdot 38^{\star *}$ & 407 & $39.07^{\star *}$ \\
\hline $12.0-12.9$ & 300 & 13.00 & 300 & 16.67 & 394 & $30 \cdot 20^{\star *}$ & 386 & $32 \cdot 64^{\star *}$ \\
\hline $13 \cdot 0-13.9$ & 300 & 9.33 & 300 & 14.00 & 410 & $33 \cdot 17^{\star *}$ & 411 & $23 \cdot 36^{\star \star}$ \\
\hline $14.0-14.9$ & 300 & $12 \cdot 00$ & 300 & $16 \cdot 33$ & 398 & $34 \cdot 67^{\star *}$ & 388 & $27 \cdot 32^{\star \star}$ \\
\hline $15 \cdot 0-15 \cdot 9$ & 300 & 11.67 & 300 & 17.67 & 420 & $29 \cdot 29^{\star \star}$ & 401 & $24.44^{*}$ \\
\hline $16 \cdot 0-16 \cdot 9$ & 300 & 12.67 & 300 & 14.33 & 423 & $26 \cdot 95^{\star \star}$ & 415 & $21.69^{*}$ \\
\hline $17.0-17.9$ & 300 & 12.33 & 300 & 9.00 & 412 & $30.58^{\star \star}$ & 403 & $16 \cdot 13^{\star \star}$ \\
\hline $18.0-18.9$ & 300 & 7.00 & 299 & $10 \cdot 37$ & 384 & $27.08^{\star *}$ & 376 & $17.02^{*}$ \\
\hline Total & 3600 & $10 \cdot 31$ & 3598 & 13.40 & 4917 & $33 \cdot 94^{\star \star}$ & 4802 & $29 \cdot 30^{\star \star}$ \\
\hline
\end{tabular}

Difference between 1995 and $2014:{ }^{*} P<0.05,{ }^{*} P<0.01$.

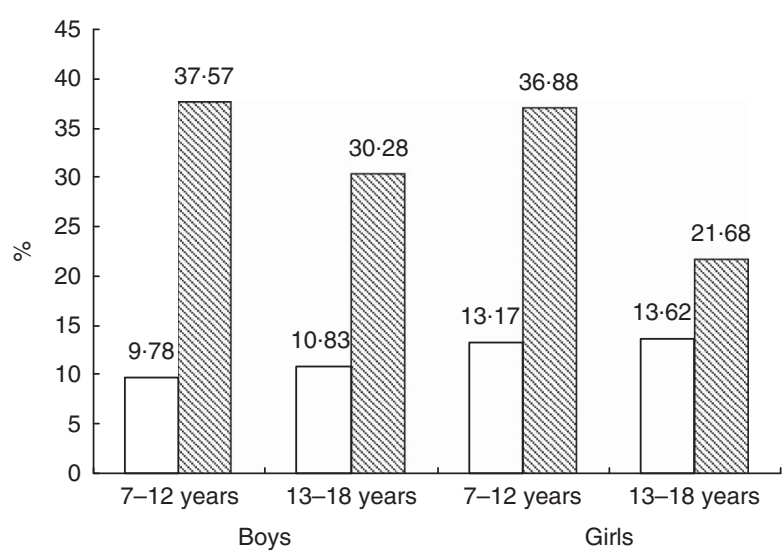

Fig. 1 Prevalence of 'high SFT' (sum of triceps and subscapular skinfold thickness above or equal to the national age- and sex-specific 85th percentile) in children (aged 7-12 years) and adolescents (aged 13-18 years), Shandong Province, China ( $n 16$ 917), in 1995 ( $\square$ ) and 2014 (因)

'Western diet', which is broadly defined by high intakes of refined carbohydrates, added sugars, fats and animalsource foods ${ }^{(14)}$. Some researchers suggested that the rapid development of the Western fast-food market (such as McDonald's and Kentucky Fried Chicken) and physical inactivity are the two major predisposing factors for increasing prevalence of childhood obesity ${ }^{(17)}$. The first Kentucky Fried Chicken and McDonald's restaurant opened in China in 1987 and 1990 respectively, and now there are more than 2000 McDonald's (http://www. mcdonalds.com.cn/index/McD/about/company) and 5000 Kentucky Fried Chicken (http://www.en.wikipedia.org/ wiki/KFC\#Asia) outlets in the country. Chinese children well recognize the images of McDonald's and Kentucky Fried Chicken and like to eat Western fast foods. Furthermore, some parents will even use these junk foods as a reward for their children. Chinese children are under great pressure to achieve scholastically, so they usually spend a lot of time doing homework instead of gymnastic exercises and sports activities ${ }^{(18,19)}$. In addition, the activity patterns of children have shifted from outdoor play to indoor entertainment: television viewing, Internet and computer games $^{(20)}$. Fortunately, the Chinese Government has realized the importance of obesity control and of playing a leading role, with the introduction of laws and regulations aimed at obesity prevention. For example, the Ministry of Health released the Nutrition Improvement Work Management Approach in 2010 to promote national nutrition initiatives and to enhance the nutrition and health status of Chinese residents ${ }^{(21)}$. A new policy of one hour of physical activity in schools every day was mandated by the Ministry of Education in 2011, which has had a beneficial effect in preventing obesity in adolescents ${ }^{(22)}$.

We also found that children (aged 7-12 years) showed a more rapid increase in the prevalence of 'high SFT' than adolescents (aged 13-18 years) between 1995 and 2014. The reason is still unclear but may be related to the difference in lifestyle and dietary patterns between the two age stages; compared with children, adolescents generally pay more attention to their body shape and engage in more physical activity ${ }^{(23)}$.

Two limitations should be noted. First, data for the present study were acquired from two independent cross-sectional surveys spanning 19 years rather than from a longitudinal cohort study, thus preventing further assessment of cohort and time effects. Second, the absence of detailed information concerning living environments, dietary patterns and physical activity at the individual level also limited our study.

\section{Acknowledgements}

Acknowledgements: The surveys on students' constitution and health were conducted under the auspices of the 
Department of Education in Shandong Province, China. The authors thank all the team members and all participants. Special thanks go to $\mathrm{Mr} \mathrm{B}$. Yu for providing access to the survey data. Financial support: This study was supported by the Medical and Health Program of Shandong Province, China (2014WS0376). The funder had no role in the design, analysis or writing of this article. Conflict of interest: There are no conflicts of interest on behalf of any of the authors. Authorship: S.-R.W. performed data analysis and drafted the manuscript, Y.-X.Z. designed the study and gave instructions on data analysis, Y.C. and M.C. helped with data collecting and sorting. All authors read and approved the final manuscript. Ethics of human subject participation: The study was approved by the Ethical Committee of the Shandong Center for Disease Control and Prevention, Shandong, China.

\section{References}

1. Wang Y \& Lobstein T (2006) Worldwide trends in childhood overweight and obesity. Int J Pediatr Obes 1, 11-25.

2. de Onis M, Blössner M \& Borghi E (2010) Global prevalence and trends of overweight and obesity among preschool children. Am J Clin Nutr 92, 1257-1264.

3. Ogden CL, Carroll MD, Kit BK et al. (2012) Prevalence of obesity and trends in body mass index among US children and adolescents, 1999-2010. JAMA 307, 483-490.

4. Ji CY \& Chen TJ (2013) Empirical changes in the prevalence of overweight and obesity among Chinese students from 1985 to 2010 and corresponding preventive strategies. Biomed Environ Sci 26, 1-12.

5. Franks PW, Hanson RL, Knowler WC et al. (2010) Childhood obesity, other cardiovascular risk factors, and premature death. $N$ Engl J Med 362, 485-493.

6. Karnik S \& Kanekar A (2012) Childhood obesity: a global public health crisis. Int J Prev Med 3, 1-7.

7. Despres JP (2012) Body fat distribution and risk of cardiovascular disease: an update. Circulation 126, 1301-1313.

8. Mei Z, Grummer-Strawn LM, Wang J et al. (2007) Do skinfold measurements provide additional information to body mass index in the assessment of body fatness among children and adolescents? Pediatrics 119, e1306-e1313.

9. Steinberger J, Jacobs DR, Raatz S et al. (2005) Comparison of body fatness measurements by BMI and skinfolds vs. dual energy X-ray absorptiometry and their relation to cardiovascular risk factors in adolescents. Int J Obes (Lond) 29, 1346-1352.

10. Addo OY, Pereira MA \& Himes JH (2012) Comparability of skinfold thickness to DXA whole-body total fat in their associations with serum triglycerides in youths. Eur J Clin Nutr 66, 989-993.

11. Zhang YX \& Wang SR (2012) Secular trends in body mass index and the prevalence of overweight and obesity among children and adolescents in Shandong, China, from 1985 to 2010. J Public Health (Oxf) 34, 131-137.

12. Zhang YX, Zhao JS, Chu ZH et al. (2016) Increasing prevalence of childhood overweight and obesity in a coastal province in China. Pediatr Obes 11, e22-e26.

13. Research Section of the Constitution and Health of Chinese Students (2007) Report on the Physical Fitness and Health Surveillance of Chinese School Students, pp. 164-189. Beijing: Higher Education Press.

14. Popkin BM, Adair LS \& Ng SW (2012) Global nutrition transition and the pandemic of obesity in developing countries. Nutr Rev 70, 3-21.

15. Du H, Bennett D, Li L et al. (2013) Physical activity and sedentary leisure time and their associations with BMI, waist circumference, and percentage body fat in 0.5 million adults: the China Kadoorie Biobank study. Am J Clin Nutr 97, 487-496.

16. Olds TS (2009) One million skinfolds: secular trends in the fatness of young people 1951-2004. Eur J Clin Nutr 63, 934-946.

17. Cheng TO (2012) Childhood obesity in modern China. Int J Cardiol 157, 315-317.

18. Tudor-Locke C, Ainsworth BE, Adair LS et al. (2007) Cross-sectional comparison of physical activity and inactivity patterns in Chinese and Filipino youth. Child Care Health Dev 33, 59-66.

19. Tudor-Locke C, Ainsworth BE, Adair LS et al. (2003) Physical activity and inactivity in Chinese school-aged youth: the China Health and Nutrition Survey. Int J Obes Relat Metab Disord 27, 1093-1099.

20. Popkin BM (2001) The nutrition transition and obesity in the developing world. J Nutr 131, issue 3, 871S-873S.

21. Wang H \& Zhai F (2003) Programme and policy options for preventing obesity in China. Obes Rev 14, Suppl. 2, 134-140.

22. Zhang YX, Zhou JY, Zhao JS et al. (2013) The role of 1-h physical activity every day in preventing obesity in adolescents in Shandong, China. Eur J Pediatr 172, 325-330.

23. Zhang J, Seo DC, Kolbe L et al. (2010) Trends in overweight among school children and adolescents in seven Chinese provinces, from 1991-2004. Int J Pediatr Obes 5 , $375-382$ 